\title{
Diseño de modelos experimentales en investigación quirúrgica
}

\author{
Del Cañizo López JF*, López Martín D*, Lledó García E**, García Barreno P*. \\ *Unidad Medicina y Cirugía Experimental. **Servicio de Urología. Hospital General Univ. Gregorio Marañón. Madrid.
}

Actas Urol Esp. 2008;32(1):27-40

\section{RESUMEN}

\section{DISEÑO DE MODELOS EXPERIMENTALES EN INVESTIGACIÓN QUIRÚRGICA}

Introducción: La investigación en Medicina se basa fundamentalmente en tres fuentes de conocimiento: En primer lugar, el hombre enfermo que es la fuente natural del conocimiento en la Clínica, en segundo lugar el cadáver, que es la fuente de conocimiento de la Anatomía Patológica y, por último, el animal de experimentación que es la fuente del conocimiento de la Fisiopatología.

Los avances experimentados en el último siglo han determinado que el concepto "animal de experimentación" deba sustituirse por un concepto mucho más amplio, el de "modelo experimental".

Objetivos: En este artículo trataremos de aclarar éste último concepto, para lo cual deberemos hacer una serie de consideraciones previas sobre el método experimental y las dos herramientas de que dispone, la estadística y el diseño de experimentos.

Palabras clave: Modelos experimentales. Investigación quirúrgica.

\section{ABSTRACT}

\section{DESIGN OF EXPERIMENTAL MODELS IN SURGICAL INVESTIGATION}

Introduction: Research in Medicine is essentially based on three knowledge resources: diseased people (natural and primary), cadaveric bodies (Pathology primary resource) and experimental animals, whom constitutes physiopathologic knowledge resource. Experimental advances reached in the last century have determined the change of the concept "experimental animal" to a wider term: "experimental model".

Objetives: This paper tryes to clarify this concept. To attain this goal, we must define previous considerations in the meaning of the concept "experimental models" and its two key-elements: statistics and design of experiments.

Keywords: Experimental models. Surgical research.

$\mathrm{L}$ a investigación en Medicina se basa fundamentalmente en tres fuentes de conocimiento: En primer lugar, el hombre enfermo que es la fuente natural del conocimiento en la Clinica, en segundo lugar el cadáver, que es la fuente de conocimiento de la Anatomía Patológica y, por último, el animal de experimentación que es la fuente del conocimiento de la Fisiopatología.

Los avances experimentados en el último siglo han determinado que el concepto "animal de experimentación" deba sustituirse por un concepto mucho más amplio, el de "modelo experimental". En este artículo trataremos de aclarar éste último concepto, para lo cual deberemos hacer una serie de consideraciones previas sobre el método experimental y las dos herramientas de que dispone, la estadística y el diseño de experimentos.

\section{EL MÉTODO EXPERIMENTAL}

El rasgo que caracteriza a una Ciencia es su Método. Las diferentes ciencias presentan, por tanto, ciertas particularidades en la utilización de los diferentes métodos.

Así como las ciencias formales (Lógica y Matemáticas) utilizan sobre todo el método deduc- 
tivo, las ciencias naturales (que pertenecen a las denominadas ciencias empíricas o experimentales) se han servido principalmente de la demostración inductiva. El método de las ciencias naturales ha sido denominado "método hipotéticodeductivo".

Los pasos del método hipotético-deductivo son:

1. Punto de partida: se detecta mediante observación y/o experimentación un problema no resuelto por el saber del que se dispone.

2. Se elaboran una o varias hipótesis explicativas del hecho observado o del problema detectado. La imaginación para formular hipótesis es indispensable en las ciencias.

3. Una vez formulada una hipótesis se deducen las posibles consecuencias contrastadas por la experiencia. Es el momento deductivo de la ciencia empírica.

4. Las consecuencias se someten a contrastación (verificación y falsación) mediante la experimentación.

- Verificación. Una hipótesis es verdadera cuando los hechos observados concuerdan con los hechos deducidos de la hipótesis.

- Falsación. Una hipótesis se refuta o "falsa" cuando los hechos no concuerdan con los hechos deducidos de la hipótesis.

5. La hipótesis, comprobada en un cierto número de casos, se acepta como ley, es decir, adquiere validez general.

El método experimental, por tanto, exige verificar las hipótesis comprobando que se repiten en un cierto número de casos, para esto cuenta con dos herramientas fundamentales: La Estadística y el Diseño experimental.

\section{LA ESTADÍSTICA Y EL DISEÑO EXPERIMENTAL}

La palabra estadística procede del vocablo estado, pues era función principal de los gobiernos de los Estados establecer registros de población, nacimientos, defunciones, cosechas, impuestos, etc. Galton (1822-1911) y Pearson (1857-1936) se pueden considerar los padres de la estadística moderna. A ellos se debe el paso de la estadística deductiva, estudiada hasta su época, a la estadística inductiva, que es la que hoy en día tiene una mayor influencia en todos los campos del saber.
Se puede definir la estadística como el estudio de los métodos y procedimientos para recoger, clasificar y analizar datos y para hacer inferencias científicas partiendo de tales datos. Tiene dos subcategorias:

- La Estadística descriptiva o deductiva, que trata de abstraer propiedades de conjuntos de observaciones mediante el empleo de métodos gráficos, tabulares o numéricos.

- La Estadística Inductiva o inferencia estadística, que trata de hacer inferencias acerca de una población o universo a partir de lo averiguado en un subconjunto del mismo denominado muestra.

La inferencia estadística proporciona una herramienta fundamental para el método científico en las ciencias naturales, de hecho, una parte muy importante de la inferencia estadística la constituye el denominado contraste de hipótesis. Las técnicas de contraste de hipótesis nos permiten rechazar o aceptar una hipótesis, la denominada hipótesis nula, con una determinada probabilidad. La probabilidad de descartar la hipótesis contrastada cuando en realidad es cierta (Error de tipo I) se denomina nivel de significancia.

Los métodos normalmente utilizados en estadística se denominan paramétricos y asumen que los datos utilizados cumplen determinadas condiciones, la principal de las cuales es que los datos se distribuyan según una distribución normal.

Cuando los datos no cumplen las exigencias del modelo paramétrico deberán utilizarse los denominados métodos no paramétricos, que ofrecen las siguientes ventajas:

1. No requieren una distribución determinada de la población de base.

2. Para tamaños de muestra muy pequeños son los que deben utilizarse a menos que se conozca con certeza la naturaleza de la distribución de la población.

3. No es necesario que los datos se asignen a una escala numérica, basta con una escala clasificatoria.

Las pruebas paramétricas son más potentes, por tanto, si se cumplen todos los supuestos del modelo paramétrico, debe elegirse una prueba paramétrica. Otra desventaja de los modelos no paramétricos es que no permiten probar interacciones dentro del modelo de análisis de varianza. 
Los métodos estadísticos asumen que los únicos errores presentes en los datos que se analizan son errores aleatorios; y todos los tests estadísticos son robustos frente a este tipo de errores. Sin embargo si el diseño de las experiencias no es adecuado, pueden introducirse en los datos errores denominados sistemáticos que los tests estadísticos son incapaces de detectar.

Las técnicas de diseño experimental, que se inician fundamentalmente en la agricultura en la que, por necesidad, los experimentos consumen mucho tiempo, tratan de optimizar los planteamientos experimentales sobre todo tratando de eliminar las fuentes de error sistemático.

Antes de definir el concepto de Diseño Experimental se deben clarificar algunos conceptos previos:

Se denominan factores de un experimento a aquellas modificaciones que introducimos en el material experimental y cuyos efectos pretendemos estudiar. Por ejemplo, podemos estar interesados en los efectos de la disminución de temperatura sobre la tensión de un anillo vascular durante un tiempo. En este caso deberemos considerar dos factores: Temperatura y Tiempo.

Es muy importante no confundir los factores con las variables que registramos para medir los rendimientos del experimento. En este caso la variable que se mide es la tensión en el anillo vascular y sus resultados son los que van a permitir deducir conclusiones acerca de la influencia de los factores en la experiencia.

Se denominan niveles a los rangos en los que se va a aplicar un determinado factor. En el ejemplo anterior podemos estar interesado en ensayar, por ejemplo, dos temperaturas: $37^{\circ}$ y $20^{\circ}$, y dos tiempos: 5 min y 10 min. Entonces el factor temperatura tendrá dos niveles: 37 y 20 , y el factor tiempo también dos niveles: 5 y 10 .

Se denomina tratamiento a una combinación de factores con un determinado nivel. En el ejemplo citado, un tratamiento posible seria: $37^{\circ}$ de temperatura y 5 minutos.

Se llama parcela o unidad experimental a aquella partida de material experimental a la que se le aplica un determinado tratamiento. En el ejemplo anterior la unidad experimental sería un anillo vascular, pero las unidades experimentales pueden ser muy variadas, desde animales completos hasta órganos aislados, porciones de tejido o células.

Se denomina bloque a un conjunto de unidades experimentales con similares características. El bloque debe tener el mismo número de unidades experimentales que de tratamientos a considerar, de tal forma que el conjunto de tratamientos se aplique a unidades experimentales que difieran entre sí lo mínimo posible, se deberán considerar tantos bloques como repeticiones de los tratamientos a realizar.

Se puede entonces definir el diseño experimental como aquel conjunto de técnicas encaminadas a estudiar y definir:

1. El conjunto de tratamientos que va a ser contrastado.

2. Las parcelas o unidades experimentales que van a utilizarse.

3. Las reglas por las cuales van a asignarse los tratamientos a las distintas unidades experimentales.

\section{Requerimientos de un buen experimento}

Ausencia de error sistemático. Hay que asegurar que las unidades que reciban un determinado tratamiento no difieren de forma sistemática de aquellas que reciben otro tratamiento, o bien que la metodología del experimento no varía de unas a otras.

Las diferencias entre tratamientos deben ser puramente aleatorias. Las unidades deben responder de forma independiente a los diferentes tratamientos. La mejor forma de suprimir el error sistemático es la aleatorización. Es fundamental considerar todas las fuentes posibles de error sistemático, ya que, como dijimos anteriormente, los tests estadísticos no son robustos ante este tipo de errores y nos podrian conducir a falsas conclusiones.

Precisión. Si se elimina el error sistemático, la estima realizada diferirá de su valor verdadero sólo por errores aleatorios. La medida de los errores aleatorios y por tanto de la precisión suele realizarse por medio del error estándar. La precisión depende de:

1. La variabilidad intrínseca del material experimental. 
2. Del número de repeticiones.

3. El diseño del experimento y del método de análisis estadístico.

Amplio rango de validez. Debe realizarse el diseño del experimento de tal forma que sus conclusiones sean aplicables a un campo 1o más amplio posible. Este requerimiento puede ser de poca importancia en el trabajo científico puro.

Simplicidad. Cuanto más sencilla sea la metodología de un experimento menos fuentes de error introduciremos en el mismo, con lo que aumentaremos su precisión y serán menores su duración y su costo.

Denominamos coste de un experimento a cualquier valor cuantitativo que exprese el esfuerzo total gastado, bien sea tiempo dinero o cantidades de materiales. El coste de un experimento es aproximadamente proporcional al número de unidades experimentales utilizadas. Puede dividirse el coste en dos partidas fundamentales:

a) Gastos de implantación.

b) Gastos por unidad experimental.

Con frecuencia hay que llegar a un compromiso entre coste y precisión, sobre todo en aquellos experimentos en los que los costes por unidad experimental son altos. En estos casos debe determinarse cuidadosamente el número de unidades a utilizar con el fin de que los costes no se disparen.

Debe realizarse un análisis previo de las fuentes por medio de las cuales se va a financiar el trabajo. En el medio hospitalario es frecuente encontrar experimentos "gratuitos".

Por su importancia se describirá someramente un tipo especial de diseños que se denominan Factoriales. Se llaman experimentos factoriales a aquellos en los que se estudian cierto número de factores simultáneamente. Cuando los tratamientos consisten en todas las combinaciones posibles que pueden formarse con los distintos niveles de los factores se dice que es un experimento factorial completo.

En un experimento factorial cada efecto principal se estima con la misma precisión que si todo el experimento se hubiera dedicado a ese factor.
Además permite estudiar interacciones entre los factores, por ejemplo adición o potenciación de los efectos.

En resumen, el método que utiliza la Fisiopatología Quirúrgica para la adquisición de conocimiento es el denominado Método Experimental o Método de las Ciencias Naturales que cuenta con dos herramientas fundamentales: La Estadística y el Diseño Experimental.

\section{MODELOS EXPERIMENTALES}

La Fisiopatología se caracteriza precisamente por tener una fuente de conocimiento propia; el animal de experimentación. Decíamos en la introducción, que el término animal de experimentación había que interpretarlo en un sentido amplio. Hoy en día el desarrollo de la Ciencia en general y de la Técnica en particular permite la utilización de otros medios además del animal de experimentación como fuentes de conocimiento en Fisiopatologia; por este motivo se debe sustituir este término por el más amplio de "Modelos Experimentales". No se debe olvidar, asimismo, que el ser humano constituye también una fuente fundamental para el conocimiento de la disciplina; es en la Clínica donde se plantean los problemas a los que la Fisiopatología debe dar explicación; y es el ser humano, considerado como sujeto experimental, en el que finalmente habrá que buscar la confirmación definitiva de nuestras hipótesis.

Según el método experimental, para considerar algo como cierto debe repetirse de forma sistemática. En muchas circunstancias y, sobre todo, en Medicina esto no es posible. Debemos recurrir entonces a modelos experimentales, lo más parecidos a la realidad, para reproducir las experiencias.

Definimos, entonces, Modelo Experimental como cualquier sistema, lógico, físico o biológico capaz de simular total o parcialmente el proceso que pretendemos estudiar.

Podemos considerar los siguientes tipos de Modelos Experimentales:

- Los modelos matemáticos (simulación por ordenador).

- Los modelos físicos o mecánicos.

- Los modelos biológicos.

- Celulares y tisulares.

- Órganos aislados.

- Animales completos. 
- El ser humano como sujeto experimental.

Como es lógico, la calidad de la información que se puede obtener de un modelo estará en relación directa con su complejidad. Normalmente la resolución de un determinado problema requiere la utilización de varios modelos, comenzando por los más sencillos como la simulación por ordenador o los modelos mecánicos, para terminar con los más complicados, como son el animal de experimentación o incluso el hombre.

\section{LOS MODELOS MATEMÁTICOS. SIMULACIÓN POR ORDENADOR}

El desarrollo espectacular que ha experimentado la informática en los últimos años ha posibilitado que herramientas, inimaginables hace tan sólo unas décadas, estén ahora disponibles prácticamente para cualquier grupo de investigación.

La situación actual permite que, la contrastación de algunas hipótesis que, anteriormente hubieran requerido el uso de animales de experimentación, hoy dia puedan contrastarse, al menos en sus estadios iniciales, por medio de programas informáticos basados en modelos matemáticos.

Así, en la actualidad, a nadie se le ocurriría iniciar la experimentación animal con una nueva válvula cardiaca, sin antes haber estudiado los patrones de flujo que pueden preverse mediante su estudio en un programa de simulación de dinámica de fluidos.

Lo mismo puede decirse para el estudio hemodinámico de un tipo de estenosis vascular, como para el estudio de la expansión de una determinada enfermedad infecciosa o del comportamiento previsto de una nueva bomba de sangre.

Es dificil prever el desarrollo de estas técnicas de simulación, ni siquiera en el futuro inmediato, pero es de esperar que en pocos años podamos disponer de programas que nos permitan simular una buena parte de los sistemas biológicos (Fig. 1).

\section{LOS MODELOS FÍSICOS O MECÁNICOS}

El siguiente escalón en la complejidad de los modelos experimentales lo constituyen los que denominamos modelos físicos, que se pueden definir como aquellos dispositivos (mecánicos, eléctricos, neumáticos, etc., o una combinación de ellos) que permiten simular en todo o en parte algún sistema biológico.

Siguiendo con el ejemplo de las válvulas cardiacas, el paso siguiente a los estudios de simulación en el ordenador será su estudio en un simulador hidrodinámico que permita visualizar el flujo real con técnicas especiales como puede ser el PIV (Particle Image Visualisation).

Una vez comprobado el perfil hidrodinámico de la válvula habrá que estudiar su resistencia mecánica con ensayos de fatiga que se realizan en los llamados duplicadores, que son sistemas en los que se somete a las válvulas a frecuencias $(1 \mathrm{pm})$ mucho más altas que las normales con el fin de acortar los ensayos que, de otra manera, deberían durar años.

Otro modelo mecánico muy utilizado, sobre todo en nuestro campo principal de investigación, es el circuito de simulación del aparato circulatorio que permite reproducir las condiciones de complianza y resistencia del árbol vascular y por tanto comprobar las características hidrodinámicas de sistemas de asistencia circulatoria antes de comenzar con las experiencias en animales (Fig. 2).

Como se puede observar, cuanto más sencillo es un modelo experimental mejor permite simular condiciones y medir parámetros que serían imposibles de implementar en modelos más complicados. Por ejemplo, no es posible visualizar el patrón de flujo de una válvula implantada en un
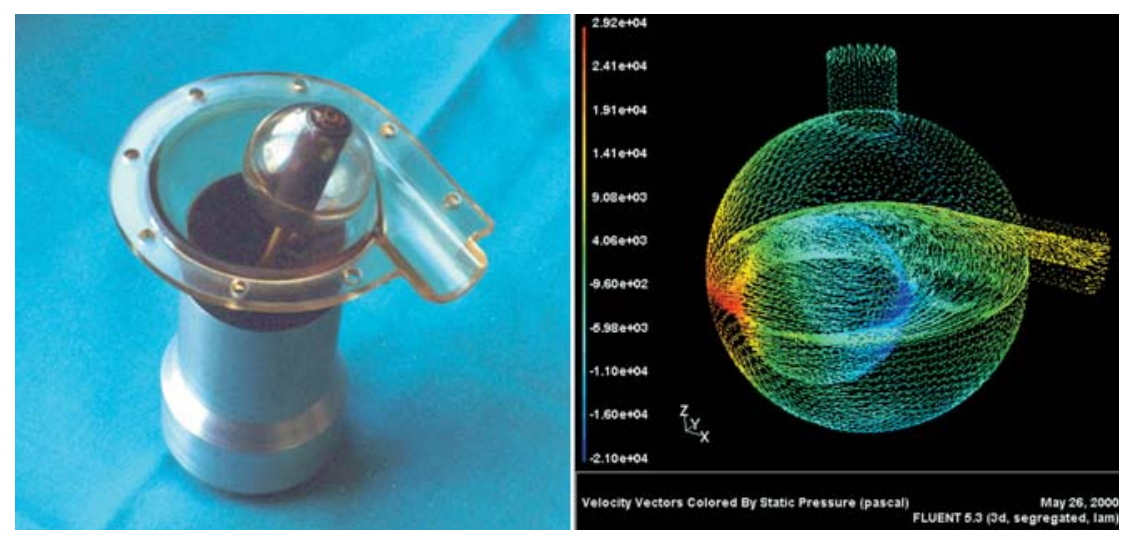

FIGURA 1. Simulación del comportamiento hidrodinámico de una bomba de bola en el ordenador. 


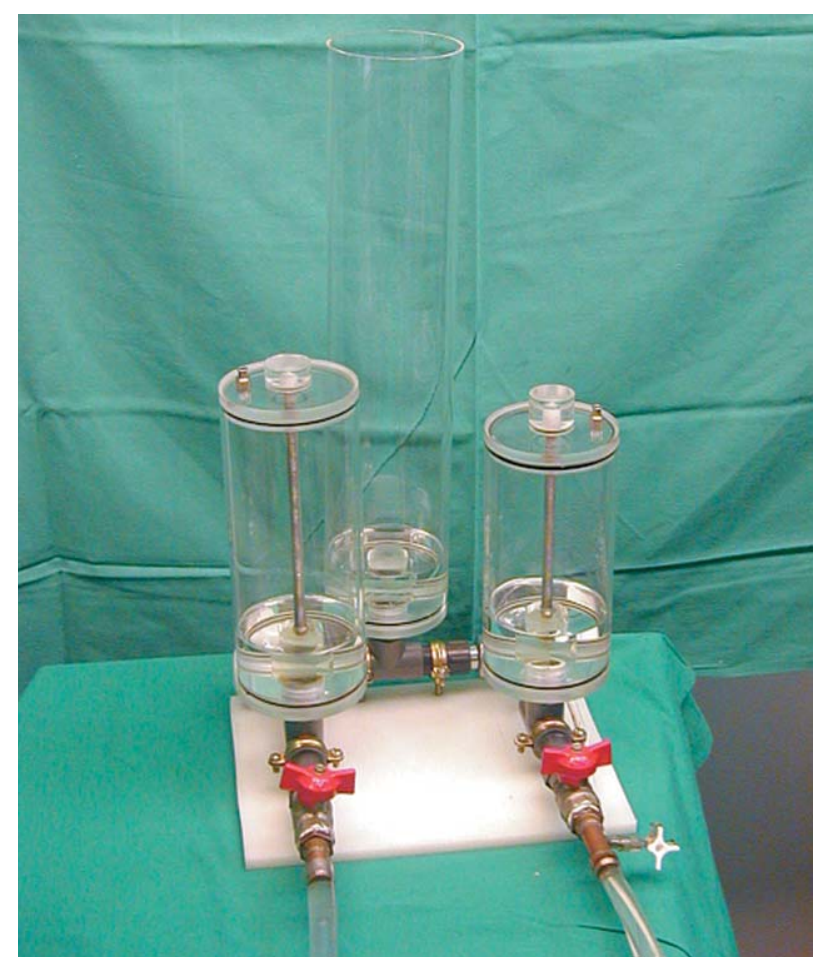

FIGURA 2. Circuito de simulación de aparato circulatorio.

animal con la precisión que se puede hacer en un simulador hidrodinámico $\mathrm{y}$, por supuesto, no se podría conseguir aumentar hasta 10 o 100 veces la frecuencia cardiaca de un animal, con el fin de comprobar la duración de una válvula, sin afectar severamente su estado circulatorio.

Sin embargo, cuanto más sencillos son los modelos más se alejan de la realidad. Es, por tanto, la gradación en la utilización de los modelos la que va a permitir obtener la máxima información sobre el proceso fisiopatológico que se pretenda estudiar.

\section{LOS MODELOS BIOLÓGICOS}

Los modelos biológicos suponen la ascensión de un nuevo escalón en la complejidad del modelo, la utilización de material biológico implica la incorporación de fenómenos homeostáticos propios de este material cuyos efectos pueden ser dificiles de controlar y predecir. La variabilidad intrínseca del material biológico es otro factor a tener en cuenta con lo que las técnicas de diseño experimental a las que nos referimos antes comienzan a ser de una importancia capital en la utilización de este tipo de modelos.
La utilización de material biológico bien sea de animales o del ser humano tiene además implicaciones de orden ético, moral y legal que habrá que tener en cuenta a la hora de planificar experimentos con estos modelos y a las que nos referiremos más adelante.

Atendiendo a su complejidad se pueden dividir en:

- Modelos celulares y tisulares.

- Órganos aislados.

- Animales de experimentación (animal completo).

\section{Los modelos celulares y tisulares}

Constituyen el escalón de menor complejidad dentro de los modelos biológicos, van desde una simple célula aislada, pasando por los cultivos de tejidos hasta la utilización de porciones de tejidos completos.

Las posibilidades de este tipo de modelos son prácticamente infinitas y dependerán fundamentalmente del campo de la fisiopatologia que estemos interesados en estudiar. Por ejemplo, la producción de óxido nítrico por la célula endotelial frente a diferentes estímulos puede estudiarse en cultivos de células endoteliales sometidos a diferentes tipos de cizallamiento en un medio líquido, la variación del tono vascular frente a diferentes condiciones de temperatura puede estudiarse con la técnica del anillo de vaso aislado, etc. (Fig. 3).

\section{Los órganos aislados}

Constituyen un modelo de gran utilidad en el estudio de muchos procesos fisiopatológicos entre los que se pueden destacar: el estudio de técnicas de conservación de órganos para trasplante, el estudio de fenómenos de isquemiareperfusión, o los efectos de diferentes variables hemodinámicas sobre la perfusión del órgano.

Sobre la utilización del animal completo tienen la ventaja de aislar el órgano de los procesos homeostáticos generales del organismo, permitiendo someterlo a condiciones que de otra manera serian imposibles de conseguir y además evitan las posibles interferencias de otros mecanismos de regulación independientes del órgano.

Implican, sin embargo, la utilización de técnicas de perfusión y de control que requieren equipamiento complejo y personal altamente especializado. 


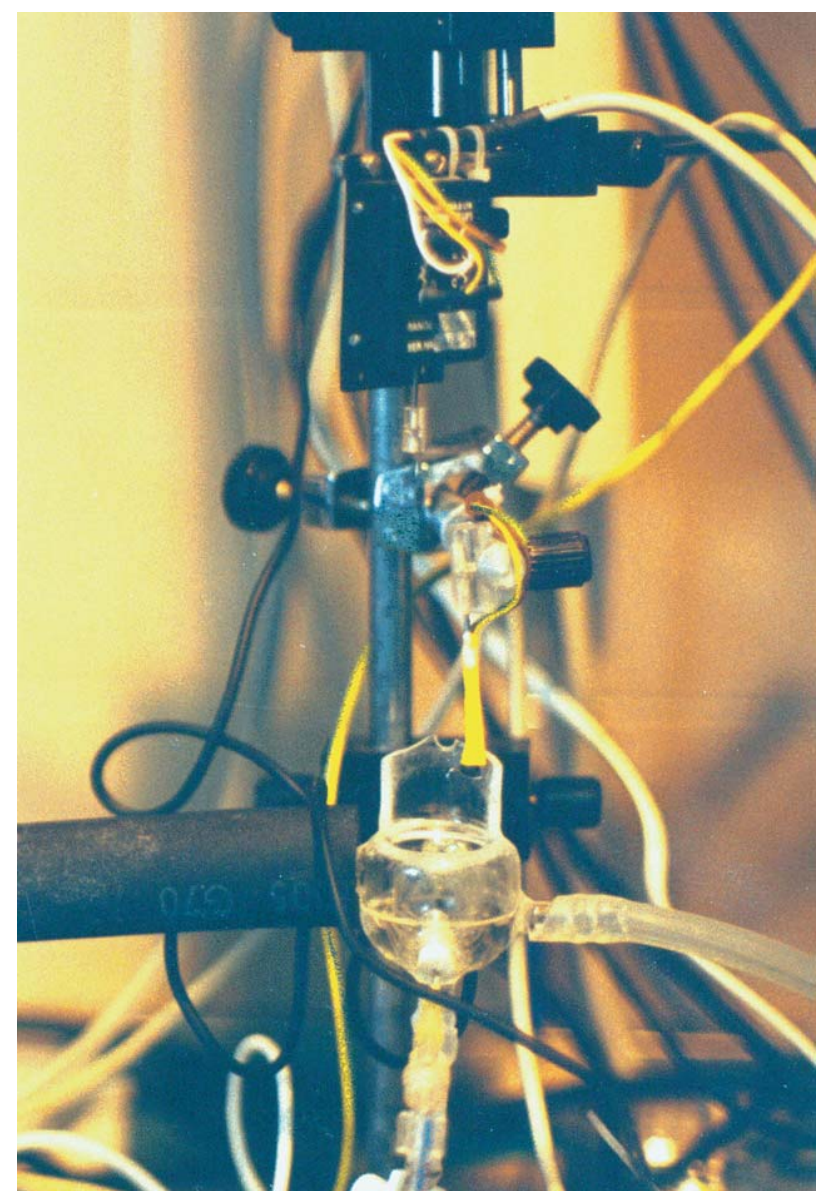

FIGURA 3. Vaso aislado en baño.

Las posibilidades de monitorización de estos modelos son también superiores en general a las que se podrian practicar en el animal completo, su principal inconveniente es precisamente el alejamiento de la realidad que pueden suponer.

Los modelos más utilizados y más ampliamente descritos son los de corazón, hígado y riñón aislados (Fig. 4).

\section{Los animales de experimentación}

Constituyen el nivel más alto de complejidad de todos los modelos experimentales y por tanto el más próximo a las condiciones reales. Son, por derecho propio, el modelo por excelencia y la fuente principal de conocimiento de la Fisiopatología Quirúrgica. Su utilización implica, sin embargo, la disponibilidad de instalaciones y personal especializados sobre todo si se pretende la utilización de animales grandes.

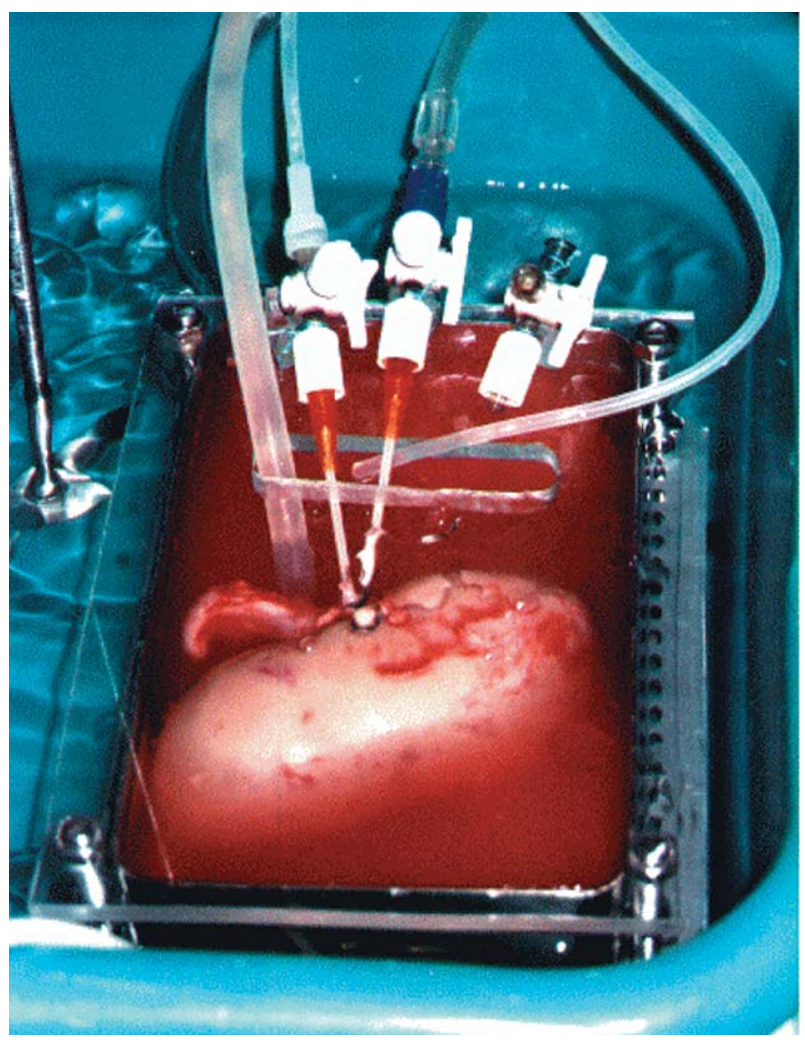

FIGURA 4. Riñón perfundido en baño.

Estas instalaciones deben incluir al menos:

- Instalaciones para la estabulación y el cuidado postoperatorio de los animales y en ocasiones para su reproducción. Es decir, animalarios correctamente equipados y atendidos por personal cualificado y calificado para esta labor.

- Instalaciones de quirófanos experimentales suficientemente equipados y atendidos por personal adecuado que permitan la realización de este tipo de experiencias con todas las garantías.

Todos los procedimientos e instalaciones relacionados con la experimentación con animales están sujetos, en nuestro país y en toda la Unión Europea, a una estricta reglamentación que resumimos en el apartado siguiente (Fig. 5).

\section{Legislación aplicable a la experimentación con seres vivos}

- DIRECTIVA DEL CONSEJO DE EUROPA $86 / 609 / \mathrm{CEE}$, sobre aproximación de las disposiciones legales, reglamentarias y administrativas de los Estados miembros respecto a protección de los animales utilizados para experimentación y otros fines científicos. 24 de Noviembre de 1986. 


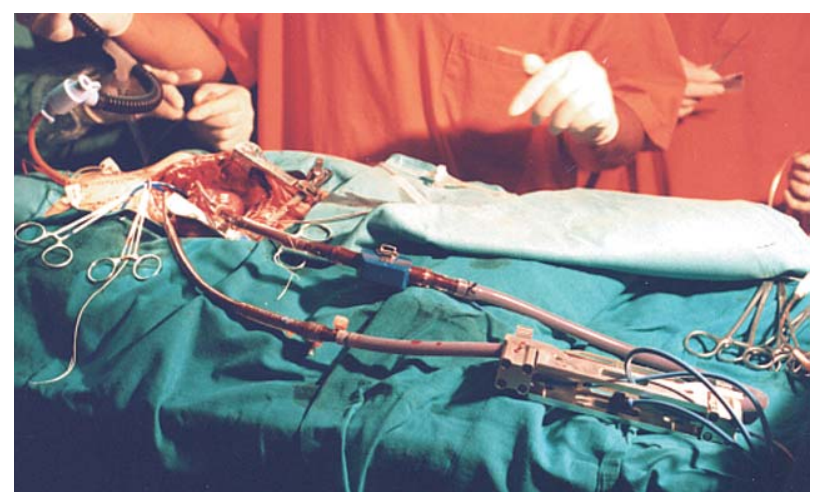

FIGURA 5. Experiencia con cerdo.

- REAL DECRETO 223/1988, de 14 de Marzo sobre protección de los animales utilizados para experimentación y otros fines científicos. B.O.E. de 18 de Marzo de 1988.

- ORDEN de 4 de Agosto de 1989 el Consejero de Agricultura y Cooperación de la C.A.M., por la que se dan normas sobre protección de los animales utilizados para experimentación y otros fines científicos. B.O.C.M. de 24 de Agosto de 1989.

- ORDEN de 13 de Octubre de 1989 por la que se establecen las normas de registro de los establecimientos de cría, suministradores y usuarios de animales de experimentación de titularidad estatal, así como las de autorización para el empleo de animales en experimentos, en desarro1lo del Real Decreto 223/1988, de 14 de Marzo. B.O.E. 18 de Octubre de 1989.

- INSTRUMENTO de Ratificación del Convenio Europeo sobre protección de los animales vertebrados utilizados con fines experimentales y otros fines científicos, hecho en Estrasburgo el 18 de Marzo de 1986. B.O.E. 25 de Octubre de 1990.

- REAL DECRETO 1201/2005, de 10 de octubre, sobre protección de los animales utilizados para experimentación y otros fines científicos. B.O.E. 21 de Octubre de 2005.

Este último Real Decreto afecta de forma muy importante a toda la experimentación con animales tanto a nivel de instalaciones como de procedimientos y, sobre todo, de acreditaciones del personal que realiza los experimentos.

\section{Consideraciones sobre la investigación con animales}

Se harán ahora una serie de consideraciones que los investigadores han de tener en cuenta a la hora de plantear experiencias con animales.
En primer lugar, los fines que puede perseguir la experimentación con animales, son:

- La prevención de enfermedades.

- El diagnóstico o tratamiento de enfermedades.

- El conocimiento de condiciones fisiológicas.

- La protección del medio ambiente.

- La investigación científica.

- La educación y formación.

- Las investigaciones forenses.

Además, los investigadores deben tener en cuenta que:

- No se deben utilizar animales si puede recurrirse a otro medio científicamente satisfactorio.

- Dentro de las posibles especies, se utilizará la más inferior en la escala filogenética.

- Se utilizarán el menor numero de animales posible y los procedimientos que causen menos dolor y sufrimiento.

- Todos los procedimientos se realizarán bajo las condiciones idóneas de anestesia y analgesia.

- Si al final del experimento el animal ha de ser sacrificado, se emplearán métodos eutanásicos humanitarios. No se mantendrá con vida a un animal si es posible que sufra dolor o angustia duraderos.

- En general, no se permitirá utilizar a un animal en más de un proyecto de investigación.

- Los animales utilizados en investigación deberán proceder de establecimientos registrados dedicados a la cría de animales de experimentación. No se utilizarán animales vagabundos de especies domésticas.

En el planteamiento de un proyecto experimental con animales es además importante tener en cuenta lo que los expertos denominan la "regla de las tres erres" tendente a que el investigador considere técnicas alternativas relacionadas con: Reemplazar los animales en la investigación, Reducir el número de animales utilizados, y/o Refinar la metodología para minimizar el dolor y el sufrimiento de los animales.

\section{Reemplazar:}

1. Utilizando otros sistemas vivos.

2. Usando Sistemas Inertes.

3. Utilizando técnicas de simulación por ordenador.

Estas consideraciones ya se hicieron anteriormente al describir los diferentes modelos experimentales. 
Reducir:

1. Animales compartidos.- Una buena organización puede permitir reducir el número de animales utilizados, coordinando los proyectos de investigación que utilizando la misma especie animal y las mismas condiciones sanitarias, dietéticas, etc, puedan compartir los animales objeto del estudio sin que esto altere los resultados finales.

2. Mejora de los diseños experimentales.- Un buen diseño experimental puede posibilitar la reducción del número de animales necesarios para conseguir los objetivos.

3. Descenso en la escala filogenética. Los animales seleccionados deberán ser de la especie menor en la escala filogenética que permita obtener resultados validos, si bien, para esta selección requerirá un estudio riguroso y un amplio conocimiento de las necesidades de la especie elegida.

4. Animales de calidad. El costo de los animales no debe hacernos olvidar la importancia de utilizar grupos uniformes de animales con condiciones idóneas: sanitarias, normas de cría, estabulación y mantenimiento. La utilización de animales de calidad garantizan la ausencia de factores desconocidos que puedan invalidar las experiencias. La utilización de animales de baja calidad pueden hacer inútil el posible sufrimiento de los animales.

\section{Refinar:}

1. Procedimientos quirúrgicos. El investigador debe utilizar los procedimientos menos agresivos para la integridad del animal de experimentación. Así como conocer y tener experiencia en las técnicas propias de la especie utilizada, como pueden las de cateterización de vías centrales.

2. Mejora en la instrumentación. Sobre todo la relacionada con la monitorización del animal (menor duración de las experiencias) y con los procedimientos de análisis de muestras que requieran menor volumen (menor tamaño del animal).

3. Mejora en el control del dolor. Los investigadores deben conocer las técnicas anestésicas y analgésicas adecuadas para la especie animal objeto del estudio.

4. Mejora en las técnicas de control. Los investigadores elaborarán protocolos de actuación precisos para prevenir el dolor y malestar de los ani- males, el veterinario responsable del Centro supervisará la idoneidad de los protocolos y se encargará de controlar la ejecución de estos.

Sensibles a la preocupación social por el bienestar de los animales de experimentación, un importante grupo internacional de investigadores formado tras el Simposio Internacional sobre "El animal de laboratorio al servicio del hombre" celebrado en Lyon (Francia) en septiembre de 1978, con motivo del centenario de la muerte de Claude Bernard, estableció en 1979 en el Centro Europeo de la Tuffs University en Taillories (Francia) los denominados "Principios de ética de la experimentación animal", que reproducimos a continuación:

Principios básicos:

Artículo 1. Los progresos del conocimiento humano son necesarios, especialmente los de la biologia, la medicina humana y la de los animales.

Artículo 2. El hombre tiene necesidad de utilizar el animal, tanto en su búsqueda del conocimiento como para nutrirse, vestirse y trabajar. De ahí el deber de respetar al animal, ente auxiliar y ser vivo como él.

Artículo 3. Toda persona que emplee animales con fines experimentales debe tener presente que están dotados de sensibilidad y memoria y son susceptibles al dolor y al sufrimiento.

Responsabilidades del experimentador:

Artículo 4. El experimentador es moralmente responsable de sus actos en el marco de la experimentación animal.

Artículo 5. Las experiencias que afecten a seres vivos y las extracciones de tejidos de sujetos vivos con fin de investigación deben ser realizadas por un científico cualificado o bajo su control directo. Las condiciones de mantenimiento de los animales de experimentación deben ser definidas y controladas por un veterinario o por un científico competente.

Artículo 6. En los ensayos que impliquen la utilización de animales debe existir una probabilidad razonable de que estos estudios contribuyan de manera importante a la adquisición de conocimientos que desembocarán eventualmente en la mejora de la salud y del bienestar del hombre y de los animales.

Artículo 7. Los métodos estadísticos, los modelos matemáticos y los sistemas biológicos in vitro 
deben ser empleados cuando sirvan para completar la experimentación animal y para reducir el número de sujetos utilizados.

Articulo 8. El experimentador debe utilizar el animal mejor adaptado a su investigación y tener en cuenta los grados sensoriales y psíquicos propios de cada especie. Los animales en peligro de extinción no deberán ser utilizados más que en circunstancias excepcionales bien definidas. Siempre que sea posible, los animales utilizados en el laboratorio provendrán de criaderos especializados que aseguren las mejores condiciones de equilibrio biológico.

Articulo 9. El experimentador debe velar para que las condiciones de mantenimiento del animal de laboratorio sean las más adecuadas y para suministrarle los cuidados necesarios antes, durante y después de las intervenciones.

Artículo 10. El experimentador tiene el deber de evitar al animal todo sufrimiento físico o psíquico inútil. Debe llevar a cabo los métodos que permitan limitar el sufrimiento y los dolores en el caso en que éstos sean inevitables.

\section{E1 ser humano como sujeto experimental}

El objetivo final de toda investigación en Medicina es su aplicación al ser humano. Por tanto cualquier procedimiento experimental, después de su correcta contrastación, siguiendo las reglas del método científico y utilizando los modelos experimentales apropiados, debe terminar por ensayarse en el hombre.

El ser humano constituye el escalón más elevado y más complejo de los modelos experimentales que hemos ido describiendo. Por sus especialísimas características y por las implicaciones éticas, morales y de toda indole que supone su utilización como sujeto experimental, el investigador debe ser especialmente riguroso en sus planteamientos.

Toda investigación que precise la utilización de seres humanos debe regirse por reglas éticas estrictas reconocidas internacionalmente adoptadas por la $18^{a}$ Asamblea Médica Mundial Helsinki, Finlandia, Junio 1964 (Declaración de Helsinki) y enmendadas por las:

- 19a Asamblea Médica Mundial, Tokio, Japón, Octubre 1975,

- 35 a Asamblea Médica Mundial, Venecia, Italia, Octubre 1983,

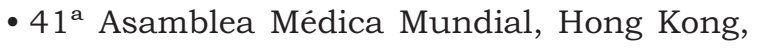
Septiembre 1989,
- y la $48^{\mathrm{a}}$ Asamblea General, Somerset West, Sudáfrica, Octubre 1996

Como dice la propia declaración, los investigadores están además obligados al cumplimiento de las leyes concretas del país donde se realice el protocolo referentes a la investigación en humanos.

\section{UN EJEMPLO PRÁCTICO}

En nuestro laboratorio hemos trabajado durante muchos años en técnicas de perfusión hipotérmica de riñón aislado con fines de preservación.

Un hecho comprobado y recogido ampliamente en la literatura es que la resistencia vascular del riñón prefundido en hipotermia desciende gradualmente durante la perfusión, éste hecho, además, se considera un signo de buena perfusión (Fig. 6).

Sin embargo, la idea que todos tenemos en cuanto a la exposición al frío, es que su efecto determina vasoconstricción. Entonces surgieron dos preguntas:

- ¿Cómo podemos explicar que el lecho vascular del riñón prefundido a bajas temperaturas (alrededor de $4{ }^{\circ} \mathrm{C}$ ) se vasodilate?

- ¿La vasodilatación se debe a algún efecto metabólico o es simplemente un efecto pasivo de la presión de perfusión sobre la pared vascular?

Obviamente, antes de plantear cualquier experiencia, tratamos de encontrar en la literatura una explicación a éste hecho y, aunque el fenómeno de la vasodilatación estaba descrito en multitud de publicaciones, nadie parecía haberse planteado el problema del por qué de dicha vasodilatación.

La posibilidad de que se debiera a un efecto metabólico vasodilatador era baja ya que a esas temperaturas $\left(4^{\circ} \mathrm{C}\right)$ muy pocos sistemas metabólicos están funcionantes. Por tanto lo primero que teníamos que comprobar era si la vasodilatación se debía a un efecto pasivo de la presión de perfusión.

Por nuestra experiencia previa con la perfusión sabíamos que el descenso mayor de las resistencias intrarrenales se producía durante la primera hora (Fig. 6). Planteamos entonces la siguiente experiencia: 


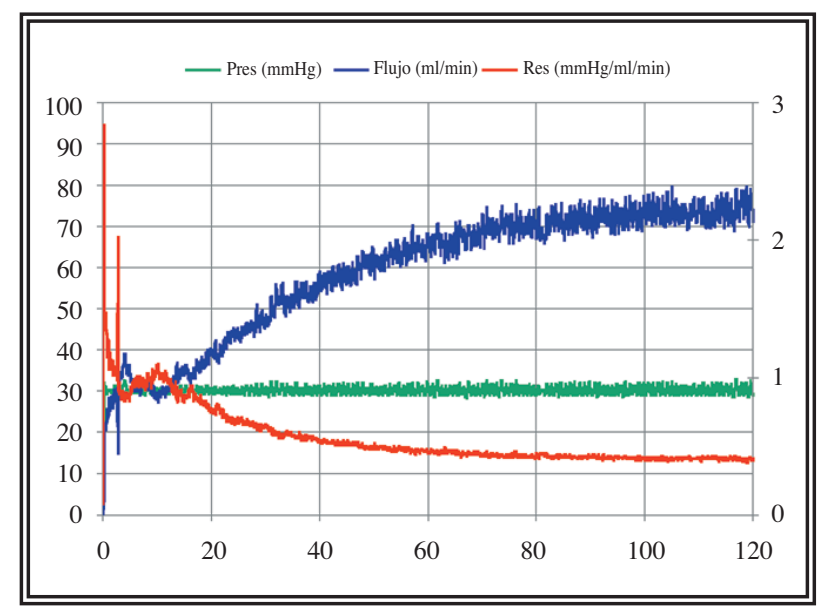

FIGURA 6. Evolución de la resistencia intrarrenal (en rojo) durante una perfusión hipotérmica a presión constante.

A los 40 minutos de una perfusión hipotérmica habitual, detenemos la perfusión durante media hora y la reiniciamos posteriormente.

$\mathrm{Si}$ el efecto se debiera a la presión de perfusión, habria que pensar que la resistencia intrarrenal cuando reiniciásemos la perfusión sería más alta o, al menos, igual que cuando la interrumpimos.

Sin embargo, cuando realizamos las experiencias observamos que le evolución de la resistencia seguía una curva similar a la de la perfusión normal como si el proceso no se hubiera interrumpido (Fig. 7).

Parecía claro, entonces, que la vasodilatación no se debía al efecto pasivo de la presión de perfusión sobre el lecho vascular renal.

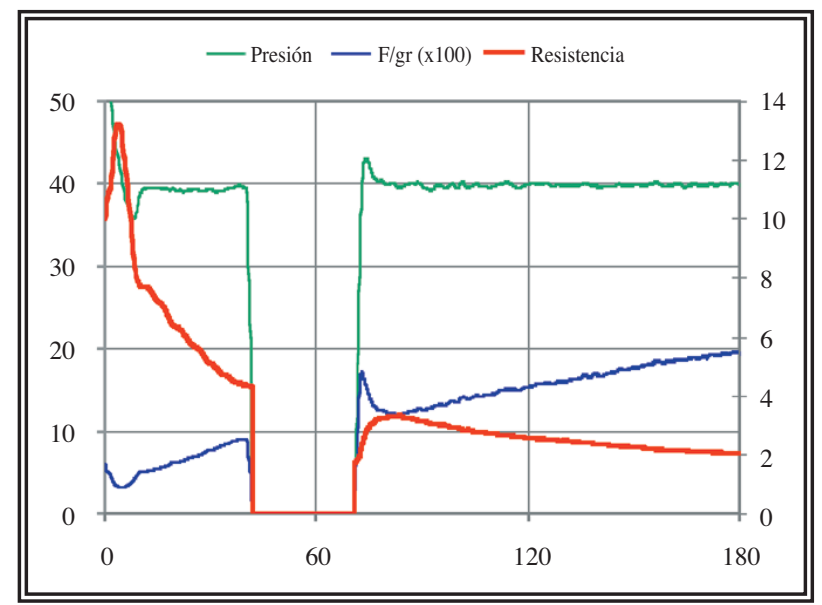

FIGURA 7. Comportamiento de la resistencia intrarrenal en una perfusión interrumpida durante $30 \mathrm{~min}$.
Debíamos pensar, por tanto, que el efecto vasodilatador se debía únicamente al descenso de la temperatura, aunque también sabíamos que si se perfundía un riñón previamente enfriado las resistencias al inicio de la perfusión eran altas.

Parecía que la vasodilatación, de alguna forma, se desencadenaba con la perfusión, pero que su mantenimiento dependía del descenso de la temperatura y no estaba ligada con el mantenimiento de la perfusión.

El modelo que estábamos utilizando hasta entonces, el riñón aislado perfundido en hipotermia, es un modelo complejo en el que intervienen multitud de sistemas metabólicos y diferentes tejidos, que podrian interferir en el fenómeno que estábamos tratando de estudiar, que, en el fondo, era el tratar de explicar por qué los vasos intrarrenales se dilataban con el descenso de la temperatura.

Decidimos entonces cambiar el modelo experimental a uno más sencillo, la técnica de vaso aislado, que se utiliza habitualmente en farmacologia. Una nueva revisión de la literatura nos indicó que, al parecer, nadie se había preocupado de estudiar el efecto de temperaturas tan bajas sobre el tono vascular.

Como el vaso más utilizado en esta técnica es la aorta de rata decidimos comenzar con este vaso. La técnica consiste en montar un anillo del vaso a estudiar entre dos ganchos metálicos fijos, uno de los cuales está unido a un transductor de fuerza, el vaso permanece introducido en un baño cuya temperatura se puede controlar. Las variaciones de tensión de la pared del vaso se traducen en cambios de la fuerza que detecta el transductor y estos cambios junto con los de temperatura se registran en un ordenador.

Los primeros estudios con la aorta de rata, que realizamos disminuyendo la temperatura del baño desde $37^{\circ}$ a $4^{\circ}$, nos mostraron que la disminución de la temperatura se acompañaba de una relajación manifiesta de la pared del vaso (Fig. 8).

Este fenómeno se repetía de forma sistemática, de forma tan evidente que llegamos a pensar incluso que podiamos tener una fuente de error sistemático que desconociamos y que no podíamos controlar. Para descartar esto realizamos el 


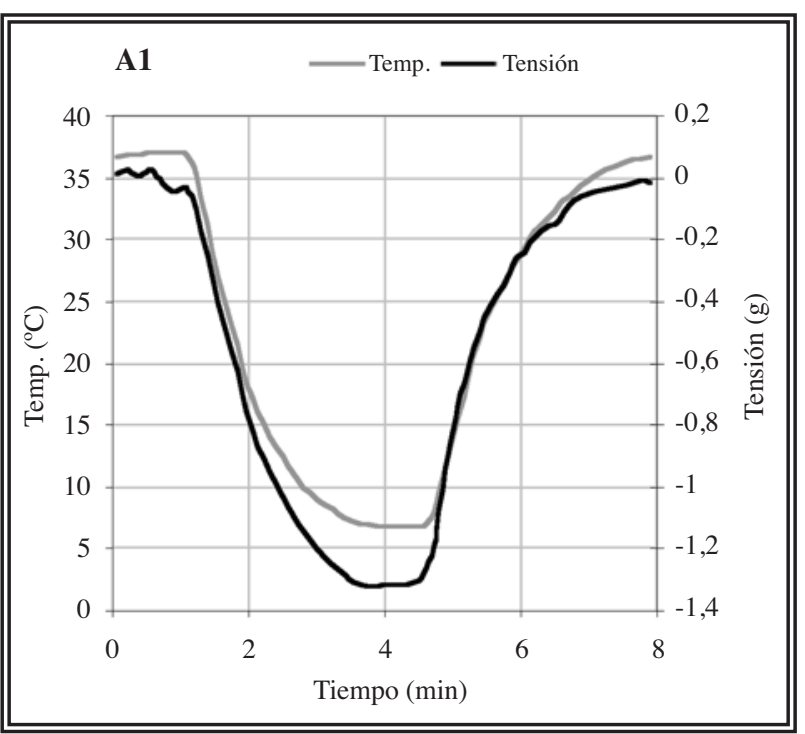

FIGURA 8. Evolución de la tensión con respecto a la temperatura de un anillo de aorta de rata.

experimento con un anillo de plástico, con el fin de descartar que el fenómeno estuviera producido por algún problema del método. Sin embargo el anillo de plástico, como era de esperar, se contraía con la temperatura (Fig. 9).

Parecía claro, entonces, que la aorta de rata se relajaba cuando la temperatura descendía. Sin embargo, nosotros queriamos aclarar la vasodilatación del lecho vascular del riñón, por tanto, decidimos repetir las experiencias utilizando arteria renal de cerdo.

Cuando ensayamos la arteria renal de cerdo, vimos que éste vaso se contraía con la temperatura (Fig. 10).

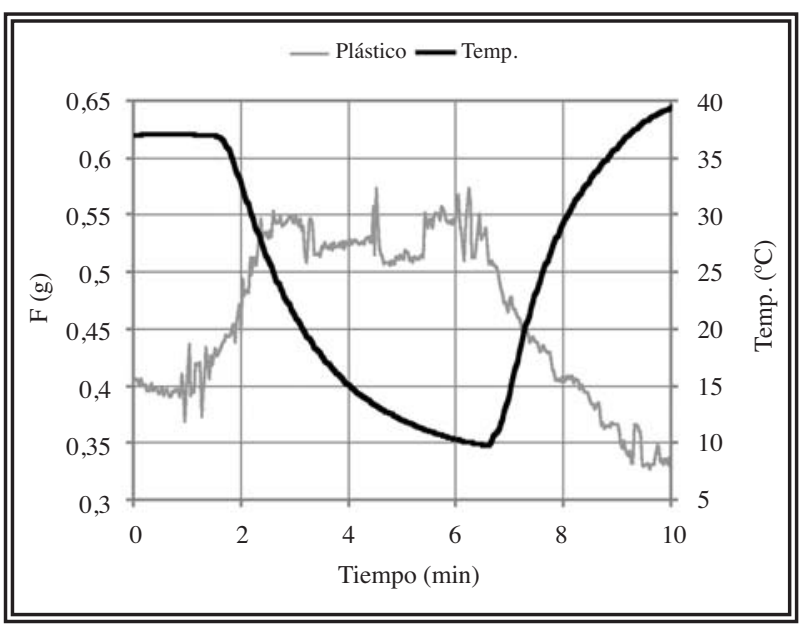

FIGURA 9. Evolución de la tensión con respecto a la temperatura de un anillo de plástico.

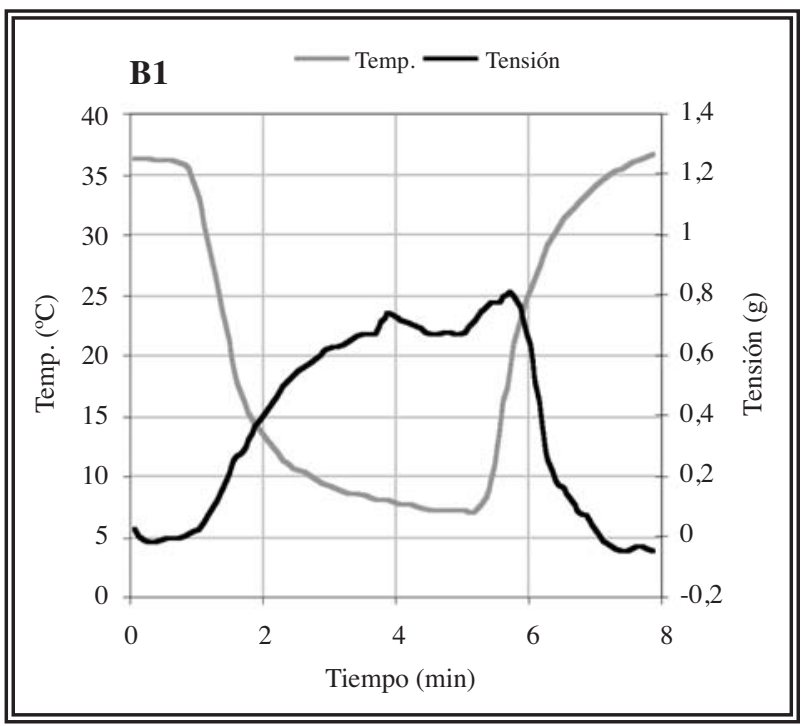

FIGURA 10. Evolución de la tensión con respecto a la temperatura de un anillo de arteria renal de cerdo.

Había que estudiar entonces el por qué del comportamiento diferente de estos dos vasos frente al descenso de la temperatura.

Estudiamos diferentes factores que pudieran influir en este comportamiento tales como: la integridad del endotelio vascular, la conservación fría del vaso o el bloqueo de la fibras musculares con un quelante del calcio.

Vimos entonces que el bloqueo de las fibras musculares y la conservación fría de los vasos inhibian la contracción de la renal de cerdo mientras que no afectaban la relajación de la aorta.

Esto nos hizo sospechar que la causa del comportamiento de la tensión de estos vasos frente al descenso de la temperatura podría deberse a la diferente estructura histológica de los mismos. La aorta de rata es un vaso fundamentalmente elástico con muy pocas fibras musculares, mientras que la arteria renal de cerdo es un vaso eminentemente muscular.

Encontramos además en la literatura que la elastina es un material peculiar que tiene un coeficiente negativo de temperatura, es de los pocos materiales que se dilata cuando la temperatura desciende.

Parecía claro entonces que la elastina podría ser la responsable de la vasodilatación de los vasos renales con el descenso de la temperatura, sin embargo este fenómeno no se reproducía claramente con la arteria renal, en algunas condi- 
ciones la contracción de la arteria con la hipotermia desaparecía pero no llegaba a dilatarse en ningún caso.

Pensamos entonces que el modelo de vaso aislado podría no reproducir fielmente las condiciones que se daban en la perfusión, de hecho, el vaso está sometido normalmente a condiciones isotónicas, esto es, la presión media en su interior es más o menos constante, sin embargo en el modelo de vaso aislado el anillo vascular está sometido a condiciones isométricas, está fijado a dos puntos fijos y lo que se mide es la tensión.

Conseguir un modelo que reprodujera las condiciones isotónicas no era fácil, decidimos entonces estudiar el comportamiento de ambos vasos frente al descenso de la temperatura en un baño y medir el tamaño del vaso con técnicas de video. En este caso el vaso no está sometido a ninguna tensión.
Comprobamos entonces que, en estas condiciones, ambos vasos se comportaban de forma similar (Fig. 11), tanto la aorta de rata como la arteria renal de cerdo disminuyen de tamaño con el descenso de la temperatura, lo que nos hace suponer que muy probablemente, la vasodilatación que experimentan los vasos renales durante la perfusión hipotérmica de riñón aislado se deba únicamente al peculiar comportamiento de la elastina frente a la temperatura y sea un fenómeno completamente pasivo.

Con este ejemplo se pone de manifiesto la importancia de los diferentes modelos experimentales en el estudio de un determinado problema y, sobre todo, que en muchas ocasiones un único modelo experimental puede no ser suficiente para su resolución.
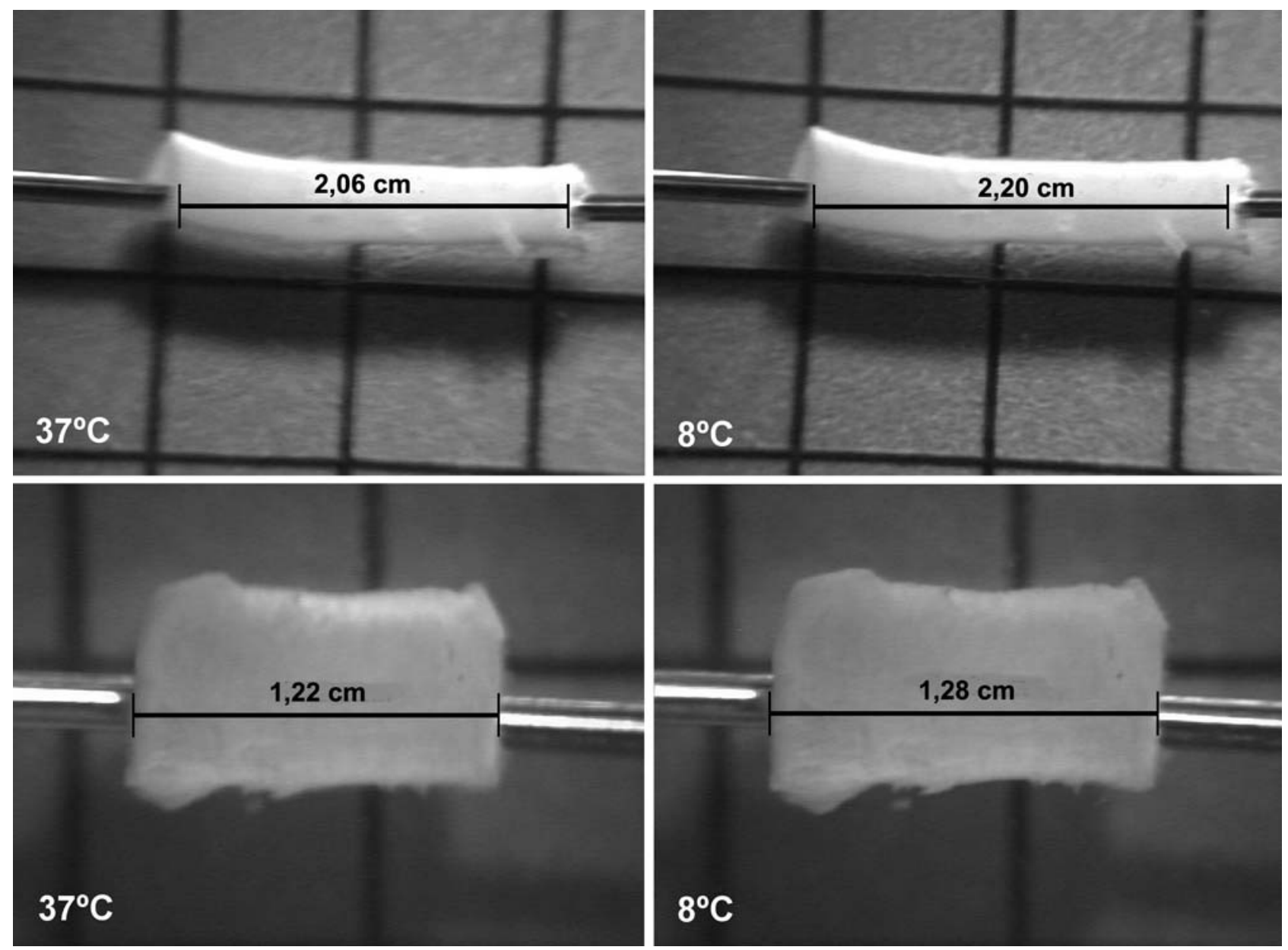

FIGURA 11. Evolución del tamaño de la aorta de rata (arriba) y la arteria renal de cerdo (abajo) con respecto a la temperatura. 


\section{UN COMENTARIO FINAL SOBRE LOS MODELOS}

Se ha visto que los interrogantes que se plantean en la Clínica Quirúrgica Humana constituyen el principal objeto de la Fisiopatologia Quirúrgica que, basándose en el método experimental, utiliza como fuente principal de conocimiento lo que se han denominado modelos experimentales.

Estos modelos experimentales se ordenan según una escala de menor a mayor complejidad hasta alcanzar al propio ser humano como sujeto de experimentación.

Cuanto más complejo es el modelo y, por tanto, más se parece a la realidad, menores grados de libertad ofrece al investigador a la hora de fijar los factores que pretende estudiar. Por tanto, a la hora de enfrentarnos con el estudio de un problema concreto, probablemente habrá que plantear diferentes modelos experimentales que nos vayan acercando a la solución final del mismo, para finalmente terminar confirmándolo en el ser humano.

La utilización del ser humano como sujeto experimental exige planteamientos tremendamente rigurosos por parte del investigador, que deben cumplir con los principios establecidos en la declaración de Helsinki y ajustarse a la legislación del país donde se realice la investigación.

\section{REFERENCIAS}

1. Remington RD, Schork MA. Estadística Biométrica y Sanitaria. Ed. Del Castillo S.A., Madrid. 1974.

2. Siegel S. Estadística no paramétrica. Ed. Trillas, México. 1979.

3. Cochran GC, Cox GM. Diseños experimentales. Ed. Trillas, México. 1980.

4. National Research Council. Guide for the care and use of laboratory animals. National Academy Press, Washington. 1997.

5. Pérez García CC, Diez Prieto I, García Partida P. Introducción a la Experimentación y Protección Animal. Secretariado de publicaciones de la Universidad de León, León. 1999.
6. Del Cañizo JF. Proyecto docente. Facultad de Medicina U. Complutense. Madrid 2001.

7. del Cañizo JF, Tejedor A, Lledó E, Radvan J, Desco M, Dulin E, et al. Isolated kidney controlled perfusion with true physiological pulsatile waveform.. Artif Organs. 1998;22(4):279-284.

8. Herrera B, Eisenberg G, Holberndt O, Desco MM, Rábano A, García-Barreno P, et al. Paradoxical effects of temperature on vascular tone. Cryobiology. 2000;41(1):43-50.

9. Herrera B, Eisenberg G, Desco MM, Holberndt O, Rábano A, Castilla M, et al. Perfusate LDH level and intrarenal resistance could not be adequate markers of perfusion quality during isolated kidney perfusion. Artif Organs. 2000;24(11): 899-902.

10. Lledó García E, Hernández Fernández C, Llorente Abarca C, del Cañizo JF. Efectos hidrodinámicos y bioquímicos de la perfusión hipotérmica de riñón aislado según el tipo de bomba utilizada. Actas Urol Esp. 2002;26(3):182-189.

11. Herrera B, Desco MM, Eisenberg G, García-Barreno P, del Cañizo JF. Role of elastic fibers in cooling-induced relaxation. Cryobiology. 2002;44(1):54-61.

12. Lledó García E, Hernández Fernández C, Llorente Abarca C, del Cañizo JF "Sistema de perfusión hipotérmica de riñón aislado controlado por ordenador.”. Actas Urol Esp 26(2):77-84 (2002).

13. Lledó García E, Hernández-Fernández C, Díez-Cordero JM, García-Barreno P, del Cañizo-López "Hydrodynamic and biochemical effects of isolated hypothermic renal perfusion depending on the pump model and perfusion solution". Transplant Proc 35(5):1661-3 (2003).

14. Lledó García E, Hernández Fernández C, MartínezSalamanca JI, Moralejo Gárate M, Cabello Benavente R, Subirá Ríos D, et al. Perfusión hipotérmica del riñón aislado: Respuesta hidrodinámica y bioquímica al perfil de aplicación de la presión. Actas Urol Esp. 2004;28(7):513522.

15. Lledó García E, Berenguer García I, Rodríguez Martínez D, Pedemonte G, Hernández Fernández C, del Cañizo López $\mathrm{JF}$. Innovaciones en la comprensión de los efectos de la isquemia fria en el injerto renal. Actas Urol Esp. 2005;29 (4):392-400.

16. Lledó Garcia E, Berenguer Garcia I, Pedemonte G, Rodríguez Martínez D, Ríos Subirá D, Hernández Fernández C, et al. Caracterización de los efectos de la isquemia normotérmica en la microcirculación renal. Actas Urol Esp. 2005;29(8):739-742.

Correspondencia autor: Dr. J.F. del Cañizo López

Unidad Medicina y Cirugía Experimental

Hospital General Universitario Gregorio Marañón

Doctor Esquerdo, 46 - 28007 Madrid

Tel.: 915868000

E-mail autor: caniño39mce.hggm.es

Información artículo: Original 